Bull. Korean Math. Soc. 48 (2011), No. 4, pp. 689-695

DOI 10.4134/BKMS.2011.48.4.689

\title{
NEIGHBORHOODS OF CERTAIN SUBCLASSES OF ANALYTIC FUNCTIONS WITH NEGATIVE COEFFICIENTS
}

\author{
Hanan E. Darwish and Mohamed K. Aouf
}

\begin{abstract}
The main object of this paper is to prove several inclusion relations associated with $(j, \delta)$-neighborhoods of various subclasses defined by Salagean operator by making use of the familiar concept of neighborhoods of analytic functions. Special cases of some of these inclusion relations are shown to yield known results.
\end{abstract}

\section{Introduction}

Let $T(j)$ denote the class of functions of the form:

$$
f(z)=z-\sum_{k=j+1}^{\infty} a_{k} z^{k} \quad\left(a_{k} \geq 0 ; j \in \mathbb{N}=\{1,2, \ldots\}\right)
$$

which are analytic in the open unit disc $U=\{z:|z|<1\}$. Following ([7] and $[10])$, we define the $(j, \delta)$-neighborhood of a function $f(z) \in T(j)$ by

$$
N_{j, \delta}(f)=\left\{g \in T(j): g(z)=z-\sum_{k=j+1}^{\infty} b_{k} z^{k} \text { and } \sum_{k=j+1}^{\infty} k\left|a_{k}-b_{k}\right| \leq \delta\right\} .
$$

In particular, for the identity function $e(z)=z$, we immediately have

$$
N_{j, \delta}(e)=\left\{g \in T(j): g(z)=z-\sum_{k=j+1}^{\infty} b_{k} z^{k} \text { and } \sum_{k=j+1}^{\infty} k\left|b_{k}\right| \leq \delta\right\} .
$$

Let $S_{j}(b, \lambda, \beta)$ denote the subclass of $T(j)$ consisting of functions $f(z)$ which satisfy the inequality

$$
\begin{gathered}
\left|\frac{1}{b}\left(\frac{z f^{\prime}(z)+\lambda z^{2} f^{\prime \prime}(z)}{\lambda z f^{\prime}(z)+(1-\lambda) f(z)}-1\right)\right|<\beta \\
(z \in U ; b \in \mathbb{C} \backslash\{0\} ; 0 \leq \lambda \leq 1 ; 0<\beta \leq 1) .
\end{gathered}
$$

Received July 20, 2009; Revised December 11, 2009.

2010 Mathematics Subject Classification. 30C45.

Key words and phrases. analytic functions, $(j, \delta)$-neighborhood, Salagean operator, complex order. 
Also let $R_{j}(b, \lambda, \beta)$ denote the subclass of $T(j)$ consisting of functions $f(z)$ which satisfy the inequality

$$
\begin{gathered}
\left|\frac{1}{b}\left(f^{\prime}(z)+\lambda z f^{\prime \prime}(z)-1\right)\right|<\beta, \\
(z \in U ; b \in \mathbb{C} \backslash\{0\} ; 0 \leq \lambda \leq 1 ; 0<\beta \leq 1) .
\end{gathered}
$$

The classes $S_{j}(b, \lambda, \beta)$ and $R_{j}(b, \lambda, \beta)$ are introduced and studied by Altintas et al. [2].

For a function $f(z) \in T(j)$, we define

$$
\begin{aligned}
& D^{0} f(z)=f(z), \\
& D^{1} f(z)=D f(z)=z f^{\prime}(z),
\end{aligned}
$$

and

$$
D^{n} f(z)=D\left(D^{n-1} f(z)\right) \quad(n \in \mathbb{N}) .
$$

The differential operator $D^{n}$ was introduced by Salagean [11]. With the help of the differential operator $D^{n}$, we say that a function $f(z) \in T(j)$ is in the class $T_{j}(n, m, \alpha)$ if and only if

$$
\operatorname{Re}\left\{\frac{D^{n+m} f(z)}{D^{n} f(z)}\right\}>\alpha \quad\left(n \in \mathbb{N}_{0}=\mathbb{N} \cup\{0\} ; m \in \mathbb{N}\right)
$$

for some $\alpha(0 \leq \alpha<1)$, and for all $z \in U$.

The operator $D^{n+m}$ was studied by Sekine [12], Aouf et al. ([4] and [5]), Hossen et al. [8] and Aouf [3]. We note that $T_{j}(0,1, \alpha)=S_{j}^{*}(\alpha)$, is the class of starlike functions of order $\alpha$ and $T_{j}(1,1, \alpha)=C_{j}(\alpha)$, is the class of convex functions of order $\alpha$ (Chatterjea [6] and Srivastava et al. [13]).

Let $S_{j}(n, m, \lambda, \gamma)$ denote the subclass of $T(j)$ consisting of functions $f(z)$ which satisfy the inequality

$$
\begin{gathered}
\left|\frac{1}{b}\left(\frac{(1-\lambda) z\left(D^{n} f(z)\right)^{\prime}+\lambda z\left(D^{n+m} f(z)\right)^{\prime}}{(1-\lambda) D^{n} f(z)+\lambda D^{n+m} f(z)}-1\right)\right|<\beta \\
\left(z \in U ; \quad b \in \mathbb{C} \backslash\{0\} ; 0 \leq \lambda \leq 1 ; 0<\beta \leq 1 ; n \in \mathbb{N}_{0} ; m \in \mathbb{N}\right)
\end{gathered}
$$

which is equivalent

$$
\left|\left(\frac{(1-\lambda) z\left(D^{n} f(z)\right)^{\prime}+\lambda z\left(D^{n+m} f(z)\right)^{\prime}}{(1-\lambda) D^{n} f(z)+\lambda D^{n+m} f(z)}-1\right)\right|<\gamma,
$$

where $\beta|b|=\gamma$.

Also let $R_{j}(n, m, \lambda, \gamma)$ denote the subclass of $T(j)$ consisting of functions $f(z)$ which satisfy the inequality

$$
\begin{gathered}
\left|\frac{1}{b}\left((1-\lambda)\left(D^{n} f(z)\right)^{\prime}+\lambda\left(D^{n+m} f(z)\right)^{\prime}-1\right)\right|<\beta, \\
\left(z \in U ; b \in \mathbb{C} \backslash\{0\} ; 0 \leq \lambda \leq 1 ; 0<\beta \leq 1 ; n \in \mathbb{N}_{0} ; m \in \mathbb{N}\right) .
\end{gathered}
$$


which is equivalent

$$
\left|\left((1-\lambda)\left(D^{n} f(z)\right)^{\prime}+\lambda\left(D^{n+m} f(z)\right)^{\prime}-1\right)\right|<\gamma
$$

where $\beta|b|=\gamma$.

We note that:

(i) $S_{j}(0,1, \lambda, \gamma)=S_{j}(\lambda, \gamma)$ and $R_{j}(0,1, \lambda, \gamma)=R_{j}(\lambda, \gamma)$;

(ii) $S_{j}(n, 1, \lambda, \gamma)=S_{j}(n, \lambda, \gamma)$ and $R_{j}(n, 1, \lambda, \gamma)=R_{j}(n, \lambda, \gamma)$ (Orhan and Kamali [9]).

\section{A set of inclusion relations involving $N_{j, \delta}(e)$}

In our investigation of the inclusion relations involving $N_{j, \delta}(e)$, we shall require Lemmas 1 and 2 below.

Lemma 1. Let the function $f(z) \in T(j)$ be defined by (1.1). Then $f(z)$ is in the class $S_{j}(n, m, \lambda, \gamma)$ if and only if

$$
\sum_{k=j+1}^{\infty} k^{n}\left[1+\lambda\left(k^{m}-1\right)\right][k-1+\gamma] a_{k} \leq \gamma .
$$

Proof. We first suppose that $f(z) \in S_{j}(n, m, \lambda, \gamma)$. Then, by appealing to the condition $(1.7)$, we readily get

$$
\operatorname{Re}\left\{\frac{(1-\lambda) z\left(D^{n} f(z)\right)^{\prime}+\lambda z\left(D^{n+m} f(z)\right)^{\prime}}{(1-\lambda) D^{n} f(z)+\lambda D^{n+m} f(z)}-1\right\}>-\gamma
$$

or, equivalently,

$$
\operatorname{Re}\left\{\frac{-\sum_{k=j+1}^{\infty} k^{n}(k-1)\left[1+\left(k^{m}-1\right) \lambda\right] a_{k} z^{k}}{z-\sum_{k=j+1}^{\infty} k^{n}\left[1+\left(k^{m}-1\right) \lambda\right] a_{k} z^{k}}\right\}>-\gamma \quad(z \in U),
$$

where we have made use of the definition (1.1).

Now choose values of $z$ on the real axis and let $z \rightarrow 1^{-}$through real values. Then the inequality (2.3) immediately yields the desired condition (2.1).

Conversely, by applying hypothesis (2.1) and letting $|z|=1$, we find that

$$
\begin{aligned}
& \left|\frac{(1-\lambda)\left(D^{n} f(z)\right)^{\prime}+\lambda z\left(D^{n+m} f(z)\right)^{\prime}}{(1-\lambda) D^{n} f(z)+\lambda D^{n+m} f(z)}-1\right| \\
& =\left|\frac{\sum_{k=j+1}^{\infty} k^{n}(k-1)\left[1+\left(k^{m}-1\right) \lambda\right] a_{k} z^{k}}{z-\sum_{k=j+1}^{\infty} k^{n}\left[1+\left(k^{m}-1\right) \lambda\right] a_{k} z^{k}}\right|
\end{aligned}
$$




$$
\begin{aligned}
& \leq \frac{\sum_{k=j+1}^{\infty} k^{n}(k-1)\left[1+\left(k^{m}-1\right) \lambda\right] a_{k}|z|^{k-1}}{1-\sum_{k=j+1}^{\infty} k^{n}\left[1+\left(k^{m}-1\right) \lambda\right] a_{k}|z|^{k-1}} \\
& \leq \frac{\gamma\left(1-\sum_{k=j+1}^{\infty} k^{n}\left[1+\left(k^{m}-1\right) \lambda\right] a_{k}\right)}{1-\sum_{k=j+1}^{\infty} k^{n}\left[1+\left(k^{m}-1\right) \lambda\right] a_{k}}=\gamma .
\end{aligned}
$$

Hence, by the maximum modulus theorem, we have $f(z) \in S_{j}(n, m, \lambda, \gamma)$, which evidently completes the proof of Lemma 1.

Similarly, we can prove the following lemma.

Lemma 2. Let the function $f(z) \in T(j)$ be defined by (1.1). Then $f(z)$ is in the class $R_{j}(n, m, \lambda, \gamma)$ if and only if

$$
\sum_{k=j+1}^{\infty} k^{n+1}\left[1+\lambda\left(k^{m}-1\right)\right] a_{k} \leq \gamma
$$

Our first inclusion relation involving $N_{j, \delta}(e)$ is given in the following theorem.

Theorem 1. Let

$$
\delta=\frac{\gamma}{(j+1)^{n-1}\left(1+\lambda\left[(j+1)^{m}-1\right]\right)(j+\gamma)} .
$$

Then

$$
S_{j}(n, m, \lambda, \gamma) \subset N_{j, \delta}(e) .
$$

Proof. For $f(z) \in S_{j}(n, m, \lambda, \gamma)$, Lemma 1 immediately yields

$$
(j+1)^{n-1}\left(1+\lambda\left[(j+1)^{m}-1\right]\right)(j+\gamma) \sum_{k=j+1}^{\infty} k a_{k} \leq \gamma,
$$

so that

$$
\sum_{k=j+1}^{\infty} k a_{k} \leq \frac{\gamma}{(j+1)^{n-1}\left(1+\lambda\left[(j+1)^{m}-1\right]\right)(j+\gamma)}=\delta
$$

which, in view of the definition (1.3), establishes the inclusion relation (2.7) of Theorem 1.

In a similar manner, by applying the assertion (2.5) of Lemma 2 instead of the assertion (2.1) of Lemma 1 to functions in the class $R_{j}(n, m, \lambda, \gamma)$, we can prove the following inclusion relationship. 
Theorem 2. If

$$
\delta=\frac{\gamma}{(j+1)^{n}\left(1+\lambda\left[(j+1)^{m}-1\right]\right)},
$$

then

$$
R_{j}(n, m, \lambda, \gamma) \subset N_{j, \delta}(e)
$$

Remark 1. (i) A special case of Theorem 1 and Theorem 2 when $n=0$ and $m=1$ was proved by Altintas et al. [2];

(ii) A special case of Theorem 1 when $b=(1-\alpha), 0 \leq \alpha<1, \lambda=n=0$ and $m=\beta=1$ was proved by Altintas and Owa [1].

\section{Neighborhoods for the classes $S_{j}^{(\alpha)}(n, m, \lambda, \gamma)$ and$$
R_{j}^{(\alpha)}(n, m, \lambda, \gamma)
$$

In this section, we determine the neighborhood for each of the classes

$$
S_{j}^{(\alpha)}(n, m, \lambda, \gamma) \text { and } R_{j}^{(\alpha)}(n, m, \lambda, \gamma)
$$

which we define as follows. A function $f(z) \in T(j)$ is said to be in the class $S_{j}^{(\alpha)}(n, m, \lambda, \gamma)$ if there exists a function $g(z) \in S_{j}(n, m, \lambda, \gamma)$ such that

$$
\left|\frac{f(z)}{g(z)}-1\right|<1-\alpha \quad(z \in U ; 0 \leq \alpha<1) .
$$

Analogously, a function $f(z) \in T(j)$ is said to be in the class $R_{j}^{(\alpha)}(n, m, \lambda, \gamma)$ if there exists a function $g(z) \in R_{j}(n, m, \lambda, \gamma)$ such that the inequality (3.1) holds true.

Theorem 3. If $g(z) \in S_{j}(n, m, \lambda, \gamma)$ and

$$
\alpha=1-\frac{\delta(j+1)^{n-1}\left(1+\lambda\left[(j+1)^{m}-1\right]\right)(j+\gamma)}{(j+1)^{n}\left(1+\lambda\left[(j+1)^{m}-1\right]\right)(j+\gamma)-\gamma},
$$

where

$$
\delta \leq(j+1)\left\{1-\gamma\left[(j+1)^{n}\left(1+\lambda\left[(j+1)^{m}-1\right]\right)(1+\gamma)\right]^{-1}\right\}
$$

then

$$
N_{j, \delta}(g) \subset S_{j}^{(\alpha)}(n, m, \lambda, \gamma)
$$

Proof. Suppose that $f(z)=z-\sum_{k=j+1}^{\infty} a_{k} z^{k} \in \mathbb{N}_{j, \delta}(g)$. We find from (1.2) that

$$
\sum_{k=j+1}^{\infty} k\left|a_{k}-b_{k}\right| \leq \delta
$$

which readily implies that

$$
\sum_{k=j+1}^{\infty}\left|a_{k}-b_{k}\right| \leq \frac{\delta}{j+1} \quad(j \in \mathbb{N}) .
$$


Next, since $g(z) \in S_{j}(n, m, \lambda, \gamma)$, we have

$$
\sum_{k=j+1}^{\infty} b_{k} \leq \frac{\gamma}{(j+1)^{n}\left(1+\lambda\left[(j+1)^{m}-1\right]\right)(j+\gamma)},
$$

so that

$$
\begin{aligned}
\left|\frac{f(z)}{g(z)}-1\right| & \leq \frac{\sum_{k=j+1}^{\infty}\left|a_{k}-b_{k}\right|}{1-\sum_{k=j+1}^{\infty} b_{k}} \\
& \leq \frac{\delta}{j+1} \cdot \frac{(j+1)^{n}\left(1+\lambda\left[(j+1)^{m}-1\right]\right)(j+\gamma)}{(j+1)^{n}\left(1+\lambda\left[(j+1)^{m}-1\right]\right)(j+\gamma)-\gamma} \\
& =\frac{\delta(j+1)^{n-1}\left(1+\lambda\left[(j+1)^{m}-1\right]\right)(j+\gamma)}{(j+1)^{n}\left(1+\lambda\left[(j+1)^{m}-1\right]\right)(j+\gamma)-\gamma}=1-\alpha,
\end{aligned}
$$

provided that $\alpha$ is given by (3.2). Thus, by the above definition, $f(z) \in$ $S_{j}^{(\alpha)}(n, m, \lambda, \gamma)$, where $\alpha$ given by (3.2). This evidently proves Theorem 3 .

The proof of Theorem 4 below is similar to that of Theorem 3 above, therefore, we omit the details involved.

Theorem 4. If $g(z) \in R_{j}(n, m, \lambda, \gamma)$ and

$$
\alpha=1-\frac{\delta(j+1)^{n}\left(1+\lambda\left[(j+1)^{m}-1\right]\right)}{(j+1)^{n+1}\left(1+\lambda\left[(j+1)^{m}-1\right]\right)-\gamma},
$$

where

$$
\delta \leq(j+1)\left\{1-\gamma\left[(j+1)^{n}\left(1+\lambda\left[(j+1)^{m}-1\right]\right)\right]^{-1}\right\},
$$

then

$$
N_{j, \delta}(g) \subset R_{j}^{(\alpha)}(n, m, \lambda, \gamma) .
$$

Remark 2. (i) A special case of Theorem 3 and Theorem 4 when $n=0$ and $m=1$ was proved by Altintas et al. [2];

(ii) Putting $m=1$ in the above results, we obtain the results obtained by Orhan and Kamali [9].

Acknowledgment. The author would like to thank the referee of the paper for helpful suggestions.

\section{References}

[1] O. Altintas and S. Owa, Neighborhoods of certain analytic functions with negative coefficients, Internat. J. Math. Math. Sci. 19 (1996), no. 4, 797-800.

[2] O. Altintas, O. Ozkan, and H. M. Srivastava, Neighborhoods of a class of analytic functions with negative coefficients, Appl. Math. Lett. 13 (2000), no. 3, 63-67.

[3] M. K. Aouf, Neighborhoods of certain classes of analytic functions with negative coefficients, Int. J. Math. Math. Sci. 2006 (2006), Art. ID 38258, 6 pp. 
[4] M. K. Aouf, H. E. Darwish, and A. A. Attiya, Generalization of certain subclasses of analytic functions with negative coefficients, Studia Univ. Babeş-Bolyai Math. 45 (2000), no. 1, 11-22.

[5] M. K. Aouf, H. M. Hossen, and A. Y. Lashin, On certain families of analytic functions with negative coefficients, Indian J. Pure Appl. Math. 31 (2000), no. 8, 999-1015.

[6] S. K. Chatterjea, On starlike functions, J. Pure Math. 1 (1981), 23-26.

[7] A. W. Goodman, Univalent functions and nonanalytic curves, Proc. Amer. Math. Soc. 8 (1957), 598-601.

[8] H. M. Hossen, G. S. Salagean, and M. K. Aouf, Notes on certain classes of analytic functions with negative coefficients, Mathematica 39(62) (1997), no. 2, 165-179.

[9] H. Orhan and M. Kamali, Neighborhoods of a class of analytic functions with negative coefficients, Acta Math. Acad. Paedagog. Nyházi. (N.S.) 21 (2005), no. 1, 55-61.

[10] St. Ruscheweyh, Neighborhoods of univalent functions, Proc. Amer. Math. Soc. 81 (1981), no. 4, 521-527.

[11] G. S. Salagean, Subclasses of univalent functions, Complex analysis - fifth RomanianFinnish seminar, Part 1 (Bucharest, 1981), 362-372, Lecture Notes in Math., 1013, Springer, Berlin, 1983.

[12] T. Sekine, Generalization of certain subclasses of analytic functions, Internat. J. Math. Math. Sci. 10 (1987), no. 4, 725-732.

[13] H. M. Srivastava, S. Owa, and S. K. Chatterjea, A note on certain classes of starlike functions, Rend. Sem. Mat. Univ. Padova 77 (1987), 115-124.

HANAN E. DARWISH

Department of Mathematics

FACULTy OF SCIENCE

MANSOURA UNIVERSITY

MANSOURA 35516, EGYPT

E-mail address: darwish333@yahoo.com

Mohamed K. Aouf

Department of Mathematics

FACULTY OF SCIENCE

MANSOURA UNIVERSITY

MANSOURA 35516, EGYPT

E-mail address: mkaouf127@yahoo.com 